\title{
Mécanisme d'action du récepteur de l'insuline : un nouveau modèle
}

Le récepteur de l'insuline est un hétérotétramère qui comporte deux types de sous-unités, $\alpha$ et $\beta$, codées par le. même gène [1]. Les deux sous-unités $\alpha$ sont extracellulaires et constituent le site de fixation de l'hormone, alors que les deux sous-unités $\beta$ sont transmembranaires et possèdent, du côté C-terminal et intracytoplasmique, un site actif de «tyrosine kinase » et des tyrosines autophosphorylables. La fixation de l'insuline sur le récepteur stimule l'activité de protéine kinase et l'autophosphorylation. Le remplacement par génie génétique des tyrosine 1162 et 1163 des sousunités $\beta$ par des phénylalanines, qui ne peuvent être phosphorylées, supprime la stimulation par l'insuline du transport de glucose ([1] et $\mathrm{m} / \mathrm{s}$ $n^{\circ} 8$, vol. 2, p. 464).

Cependant, certains résultats permettent de remettre en question le dogme selon lequel la stimulation de l'activité kinasique et l'autophosphorylation du récepteur sont indissociablement liés à la transmission des effets métaboliques de l'insuline. Certains anticorps monoclonaux qui se fixent sur la sous-unité $\alpha$ en un site distinct de celui de l'insuline stimulent le transport de glucose sans stimuler l'activité kinasique de la sousunité $\beta[2,3]$. Ces anticorps seraient même actifs sur des récepteurs dont le site de fixation de l'ATP a été muté [3].

Ce sont des résultats de même nature qui ont été obtenus par des équipes françaises de Nice et de Paris $[5,6]$. Utilisant la séquence d'ADN codant pour le récepteur muté au niveau des tyrosines phosphorylables, résidus 1162 et 1163, Anne Debant et ses collaborateurs ont tout d'abord démontré que ce type de mutation ne bloquait pas l'induction par l'insuline $\mathrm{m} / \mathrm{s} n^{\circ} 2$ vol. 5 , féurier 89 d'une réponse proliférative des cellules synthétisant le récepteur modifié, alors que, comme cela avait été démontré auparavant, la réponse métabolique à l'hormone était perdue [5].

Mieux même, il était possible de reproduire une réponse métabolique (la stimulation de la synthèse de glycogène) en utilisant des anticorps de lapin anti-récepteurs qui étaient seulement moitié moins efficaces sur le récepteur muté que sur le récepteur normal [6].

Les fragments Fab des anticorps (c'est-à-dire les régions variables dépourvues du fragment Fc de la chaîne lourde et donc des ponts disulfures qui maintiennent reliés les deux moitiés d'un anticorps) sont inactifs, mais la stimulation est obtenue lorsque, après traitement des cellules par ces fragments Fab spécifiques, on rajoute des immunoglobulines complètes anti-Fab de lapin (figure 1).

La différence entre les fragments Fab d'une part, les anticorps complets ou bien le mélange fragments Fab/antiFab d'autre part, est que seule les seconds réactifs sont «divalents » et peuvent donc aboutir à l'agrégation des molécules de récepteur. Il a été montré depuis longtemps que la liaison des ligands sur leurs récepteurs induisait le regroupement de ceux-ci en certaines zones de la membrane qui donneront naissance aux « invaginations mantelées » (coated pits) ; cette agrégation des récepteurs serait notamment indispensable aux effets de l'insuline [7]. On peut don' faire l'hypothèse que, dans les conditions normales, le principal rôle de l'autophosphorylation des tyrosines 1162 et 1163 est de participer à, peut-être de déclencher, l'agrégation des récepteurs qui serait requise pour lá trans- mission du message hormonal. Il se pourrait d'ailleurs que cette séquence "autophosphorylation-agrégation », soit nécessaire mais non suffisante. En effet, une délétion des 43 acides aminés carboxyterminaux de la sousunité $\beta$ (figure 1, page 118) n'interfère pas avec la stimulation par l'insuline de l'autophosphorylation du récepteur; l'internalisation normale de ce récepteur modifié suggère aussi que l'agrégation est normale $[4,8]$. Cependant, ces récepteurs tronqués ne permettent plus la transmission du message hormonal [9], ce qui semble indiquer que ces acides aminés interviennent d'une manière ou d'une autre dans la stimulation des voies métaboliques sensibles à l'insuline.

Le modèle de l'activité du récepteur que l'on peut, aujourd'hui, proposer est ainsi le suivant: la fixation de l'insuline sur la sous-unité $\alpha$ provoque un changement conformationnel aboutissant à la stimulation de l'activité de tyrosine kinase et à l'autophosphorylation de la sousunité $\beta$. Autophosphorylation et stimulation de l'activité kinasique sont d'ailleurs étroitement liées, la seconde ne se produisant normalement pas si les tyrosines autophosphorylables sont modifiées* (note p. 118).

Les récepteurs phosphorylés s'agrègent, ce qui permet une stimulation des voies métaboliques... peut-être, par exemple, par l'intermédiaire de l'activation d'une phospholipase et de la libération d'un inositol-glycane, second messager intracellulaire $\left(\mathrm{m} / \mathrm{s} n^{\circ} 1\right.$, vol. 3, p. 54). L'extrémité C-terminale de la sous-unité $\beta$ interviendrait dans cette activation. Il faut remarquer que, selon ce modèle, le seul substrat physiologique de l'acti- 


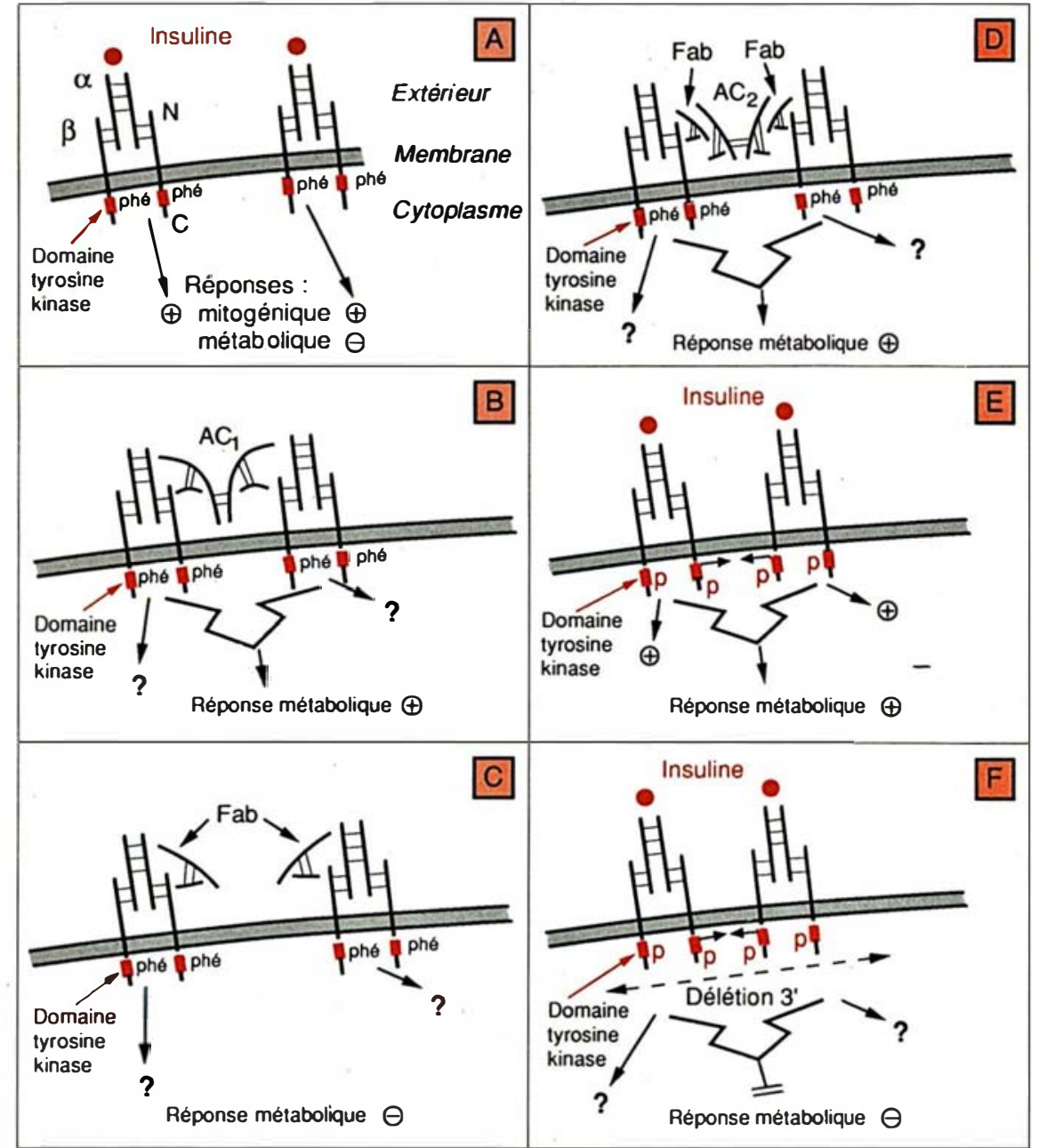

Figure 1. Activité de récepteurs de l'insuline normaux et modifiés. A. En cas de modification des tyrosines 1162 et 1163 (mutées en phénylalanine), la fixation de l'insuline sur le récepteur ne peut activer l'autophosphorylation des. tyrosines, ne stimule pas l'activité de tyrosine kinase de la sous-unité $\beta$ et ne permet pas de transmettre à la cellule le message métabolique de l'insuline. En revanche, le message mitogénique est transmis. B. Des anticorps complets (bivalents) antirécepteurs $\left(A C_{1}\right)$ permettent d'induire une réponse métabolique même quand les récepteurs de l'insuline sont mutés au niveau des tyrosines 1162 et 1163 . C. Cette réponse métabolique est perdue lorsque les anticorps $A C$, sont remplacés par des fragments monovalents Fab... D. ... mais elle est rétablie lorsque la bivalence, et donc l'agrégation des récepteurs, est obtenue grâce à l'addition d'anticorps complets anti-fragments Fab $\left(A C_{2}\right)$. E. Au niveau du récepteur normal, la fixation de l'insuline stimule l'autophosphorylation et l'activité de tyrosine kinase de la sousunité $\beta$, ce qui aboutirait à l'agrégation des récepteurs. F. En cas de délétation de l'extrémité $C$-terminale de la sous-unité $\beta$, l'autophosphorylation et, probablement, l'agrégation des récepteurs induits par l'insuline ne sont pas modifiées. Cependant, la transmission du message métabolique est interrompue.

* Il se pourrait néanmoins qu'existât une activité tyrosine kinase active sur certains susbtrats
et indépendante de l'autophosphorylation. Elle pourrait expliquer le maintien de l'effet 118 vité kinasique de la sous-unité $\beta$ serait la sous-unité $\beta$ elle-même. Par ailleurs, l'activité mitogénique pourrait ne pas exiger l'agrégation préalable des récepteurs. Avec beaucoup d'humilité, reconnaissons cependant qu'il persiste beaucoup d'incertitudes quant au mode de transmission du message insulinique, le modèle proposé ci-dessus n'étant peut-être que temporaire!

Axel Kahn

\section{RÉFÉRENCES}

1. Clauser E. Le récepteur de l'insuline, second messager de l'hormone. médecinel sciences $1988 ; 4$ : 72-82.

2. Forsayeth JR, Montemurro A Maddux BA, De Pirro R, Goldfine ID. Effect of monoclonal antibodies on human insulin receptor autophosphorylation, negative cooperativity and down-regulation. J Biol Chem 1987; 262 : $4134-40$

3. Espinal J. What is the role of the insulin receptor tyrosine kinase ? Trends Biochem Sci $1988 ; 13: 367-8$.

4. Carpentier JL. Internalisation et recyclage du récepteur de l'insuline. médecine/sciences $1988 ; 4: 83-9$.

5. Debant A, Clauser E, Ponsio G, et al. Insulin receptors mutated on tyrosines 1162 and 1163 which fair to display the insulin effects on glucose metabolism do transmit the mitogenic effects of the hormone. Proc Natl Acad Sci USA 1988 ; 85 : 8032-6.

6. Debant A, Ponzio G, Clauser E, Contreres JO, Rossi B. Receptor cross-linking restores an insulin metabolic effect altered by mutation on tyrosine 1162 and tyrosine 1163. Biochemistry $1989 ; 28: 14-7$.

7. Kahn CR, Baird KL, Jarret BD, Flier JS Direct demonstration that receptor cross-linking or aggregation is important in insulin action. Proc Natl Acad Sci USA 1978; 75 : 4209-13.

8. McClain DA, Maegawa H, Levy J, et al. Properties of a human insulin receptor with a COOH-terminal truncation. I : insulin binding, autophosphorylation and endocytosis. J Biol Chem 1988 ; 263 : 8904-11.

9. Maegawa $\mathrm{H}$, McClain DA, Freidenberg G et al. Properties of a human insulin receptor with a $\mathrm{COOH}$-terminal truncation. Il truncated receptors have normal kinase activity but are defective in signaling metabolic effects. J Biol Chem 1988; 263 : 8912-7. 